\title{
Pairing Symmetry and Multiple Energy Gaps in Multi-Orbital Iron-Pnictide Superconductors
}

\author{
Liang-Jian Zou and Feng Lu \\ Key Laboratory of Materials Physics, Institute of Solid State Physics, \\ the Chinese Academy of Sciences \\ People's Republic of China
}

\section{Introduction}

Since the new high-Tc superconducting family based on iron pnictides was discovered Kamihara et al. (2008); Chen et al. (2008a;b), and the critical temperature was lifted to $56 \mathrm{~K}$ under high pressure Wu et al. (2009) which is considerably larger than the McMillan Limit McMillan (1968), the superconductive pairing mechanism and properties have attracted great interests experimentally and theoretically. These newly-found superconductors also provide a potential application prospect in two respects: on the one hand, simple components and rich resource of the FeAs-based compounds show the most possibility of large-scale applications; on the other hand, extremely large upper critical fields $\mathrm{H}_{c 2}$ Wen et al. (2008) in FeAs superconductors imply realistic applications in the near future. To find higher $\mathrm{T}_{c}$ FeAs superconductors in further experiments and to improve their critical currrent density, it is essentially important to theoretically understand various normal and superconducting properties of iron pnictides, especially the superconducting pairing symmetry and its microscopic pairing mechanism. Once the pairing symmetry is known, many superconducting properties could be qualitatively understood.

Soon after the finding of the superconductivity in $\mathrm{LaFeAsO}$, the first-principles electronic structure calculations Mazin et al. (2008); Boeri et al. (2008); Cao et al. (2008); Ma et al. (2010) showed that Fe $3 d$ orbits contribute the major spectral weight near the Fermi surface: the Fermi surface of $\mathrm{LaFeAsO}$ consists of two hole-type circles around the $\Gamma$ point and two electron-type co-centered ellipses around the $M$ point Zhang et al. (2009), indicating that the multi-orbital character is important in the FeAs superconductors. This is in a sharp contrast to the single-orbital character of high- $\mathrm{T}_{C}$ cuprate. In these multi-orbital systems, one of the central problems is the superconducting pairing mechanism and its pairing symmetry. In fact, shortly after the determination of crystal structures of F-doped LaFeAsO, several authors proposed the constraint of the superconducting pairing symmetry of iron pnictides by applying the symmetric operations of the crystal point group on the pairing wavefunctions Mazin et al. (2008). However, such an analysis does not consider the Fermi surface topologies and the pairing potential of the superconductivity, hence can not solely determine the pairing symmetry in superconducting $\mathrm{LaFeAsO}_{1-x} \mathrm{~F}_{x}$ Lin et al. (2008).

Meanwhile, based on microscopic spin fluctuation interactions in $\mathrm{LaFeAsO}$, some authors proposed various superconducting pairing symmetries range from spin singlet $d$-wave Mu et al. (2008); Millo et al. (2008), s-wave Wang et al. (2009a;b); Nomura et al. (2008), the 
mixture of $S_{x^{2} y^{2}}$-wave and $d_{x^{2}-y^{2}}$-wave Seo et al. (2008) to spin triplet $p$-wave Dai et al. (2008); Lee et al. (2008). These suggestions on the pairing symmetry raised critical and hot debates in the literature. A few authors focused on the microscopic origin of the superconducting pairing according to the antiferromagnetic spin fluctuation and the Fermi surface nesting topology through the characteristic wavevector $\mathbf{Q}=(\pi, 0)$ of the antiferromagnetic spin fluctuations. They proposed the $\mathrm{s}_{+-}-$-wave pairing symmetry Mazin et al. (2008); Kuroki et al. (2008), i.e. the phase of the superconducting order parameters of the inner Fermi surface around the $\Gamma$ point is antiphase to that of the Fermi surface around the $M$ point. The $\mathrm{s}_{+-}$-wave symmetry of the superconducting order parameters seems to receive sufficient support in theory and experiment Mazin et al. (2008), and is consistent with the nesting picture of electron-type and hole-type Fermi surfaces in FeAs-based superconductors. However, the most recently found $\mathrm{K}_{x} \mathrm{Fe}_{2-y} \mathrm{Se}_{2}$ compounds clearly rule out the presence of a hole-type Fermi surface around the $\Gamma$ point Xiang et al. (2011), suggesting that an alternative pairing symmetry is possible. Actually, from the researching history of the high- $\mathrm{T}_{\mathcal{C}}$ cuperates, we have known that a pairing mechanism based on the Fermi surface nesting is rather delicate, since any finite electron-electron interaction, which usually occurs in high- $\mathrm{T}_{c}$ cuprates and iron pnictides, will destroy the perfect nesting of the Fermi surfaces. These disagreements and debates in the experimental data and theoretical results on the superconducting pairing symmetry of iron pnictides appeal for more efforts to unveil the mysterious nature of the superconducting iron pnictides.

On the other hand, the effect of the electron correlation in iron pnictides should be taken into account, since the bad metallic behavior and the existence of antiferromagnetic spin moments suggest that the iron pnictide is close to a metal insulator transition Haule et al. (2008). In this Chapter, starting with the minimal two-orbital t-t'-J-J' model Manousakis et al. (2008); Raghu et al. (2008), we develop a mean-field theory of the multi-orbital superconductors for the weak, intermediate, and strong correlation regimes, respectively. Taking a concrete $t-\mathrm{t}^{\prime}-\mathrm{J}-\mathrm{J}^{\prime}$ model which has the same topology as the Fermi surface and the band structures of LaFeAsO, we obtain the superconducting phase diagram, the quasiparticle spectra in normal state and superconducting phase, and the ARPES manifestation of the superconducting energy gaps. Our theory is applicable not only for FeAs superconductors, but also for ruthenate and heavy fermion, and other spin-fluctuation mediated multi-orbital superconductors. For realistic iron pnictides, we show that the pairing symmetry $d_{x^{2}-\eta y^{2}}+S_{x^{2} y^{2}}$-wave is stable in the reasonable parameters region; two superconducting gaps and their weak anisotropy and nodeless qualitatively agree with the observations in ARPES experiments. However, a quantitative comparison between theory and experiment shows a more elaborate theoretical model is necessary.

The rest of this Chapter is arranged as follows: in Sec.II we present the theory and methods for multi-orbital superconductivity; in Sec.III and IV we show the numerical results on the pairing symmetry of multi-orbital iron-pnictide superconductors, and the orbital dependence of superconducting energy gaps; Sec.V is devoted to the comparison between our theory and experimental observations, and finally we make a concluding remarks in Sec.VI.

\section{Theory and methods of multi-orbital superconductivity}

For the iron-pnictide compounds, the electron-phonon coupling seems to be irrelevant to the superconducting pairing origin Boeri et al. (2008), the antiferromagnetic spin fluctuation is 
naturally thought to contribute the pairing glue of the superconducting electron pairs, due to the antiferromagnetic ground state in updoped FeAs compounds. Considering the multi-band and electron correlation characters, a minimal model for describing the low-energy physics of the FeAs-based superconductors is the two-orbital t-J model and its extension. Based on the band structures results and theoretical analysis, the twofold-degenerate $d_{x z} / d_{y z}$ orbits are essential for the ironpnictide superconductors. We firstly depict such physical processes with the two-orbital Hubbard model,

$$
\begin{aligned}
\hat{H}= & \sum_{<i j>\alpha \beta \sigma} t_{i j}^{\alpha \beta} \hat{c}_{i \alpha \sigma}^{\dagger} \hat{c}_{j \beta \sigma}+\sum_{\ll i j \gg \alpha \beta \sigma} t_{i j}^{\prime \alpha \beta} \hat{c}_{i \alpha \sigma}^{\dagger} \hat{c}_{j \beta \sigma} \\
& +U \sum_{i \alpha} \hat{c}_{i \alpha \uparrow}^{\dagger} \hat{c}_{i \alpha \uparrow} \hat{c}_{i \alpha \downarrow}^{\dagger} \hat{c}_{i \alpha \downarrow}+U^{\prime} \sum_{i \sigma \sigma^{\prime}} \hat{c}_{i 1 \sigma}^{\dagger} \hat{c}_{i 1 \sigma} \hat{c}_{i 2 \sigma^{\prime}}^{\dagger} \hat{c}_{i 2 \sigma^{\prime}} \\
& -J_{H} \sum_{i \sigma \sigma^{\prime}} \hat{c}_{i 1 \sigma}^{\dagger} \hat{c}_{i 1 \sigma^{\prime}} \hat{c}_{i 2 \sigma^{\prime}}^{\dagger} \hat{c}_{i 2 \sigma} \\
& +J_{H} \sum_{i \alpha \neq \alpha^{\prime}} \hat{c}_{i \alpha \uparrow}^{\dagger} \hat{c}_{i \alpha^{\prime} \uparrow} \hat{c}_{i \alpha \downarrow}^{\dagger} \hat{c}_{i \alpha^{\prime} \downarrow}
\end{aligned}
$$

where $c_{i \alpha \sigma}^{\dagger}$ creates a $d_{x z}(\alpha=1)$ or $d_{y z}(\alpha=2)$ electron with orbit $\alpha$ and spin $\sigma$ at site $\mathrm{R}_{i} . \mathrm{t}$ and $t^{\prime}$ denotes the hopping integrals of the nearest-neighbor (NN) and the next-nearest-neighbor (NNN) sites, respectively. $\mathrm{U}, \mathrm{U}^{\prime}$ and $\mathrm{J}_{H}$ are the intra-orbital, inter-orbital Coulomb interactions and the Hund's coupling.

In the strongly correlated regime, it is well known that the t- $\mathrm{t}^{\prime}-\mathrm{J}-\mathrm{J}^{\prime}$ model can be derived from Eq.(1); meanwhile, even in the weak correlation regime in the atomic limit Manousakis et al. (2008), the two-orbital Hubbard model in Eq.(1) can derive to the t- $\mathrm{t}^{\prime}-\mathrm{J}-\mathrm{J}^{\prime}$ model, although not strictly. Thus, we can describe the low-energy processes in iron pnictides with the two-orbital $\mathrm{t}-\mathrm{t}^{\prime}-\mathrm{J}-\mathrm{J}^{\prime}$ model,

$$
H=H_{t-t^{\prime}}+H_{J-J^{\prime}}
$$

on a quasi-two-dimensional square lattice. This Hamiltonian consists of the tight-binding kinetic energy $H_{t-t^{\prime}}$ and the interaction part $H_{J-J^{\prime}}$. The kinetic energy term reads,

$$
H_{t-t^{\prime}}=\sum_{k \sigma}\left[\left(\varepsilon_{k x z}-\mu\right) c_{k 1 \sigma}^{\dagger} c_{k 1 \sigma}+\left(\varepsilon_{k y z}-\mu\right) c_{k 2 \sigma}^{\dagger} c_{k 2 \sigma}+\varepsilon_{k x y}\left(c_{k 1 \sigma}^{\dagger} c_{k 2 \sigma}+c_{k 2 \sigma}^{\dagger} c_{k 1 \sigma}\right)\right]
$$

with the notations

$$
\begin{aligned}
& \varepsilon_{k x z}=-2\left(t_{1} \cos k_{x}+t_{2} \cos k_{y}+2 t_{3} \cos k_{x} \cos k_{y}\right), \\
& \varepsilon_{k y z}=-2\left(t_{2} \cos k_{x}+t_{1} \cos k_{y}+2 t_{3} \cos k_{x} \cos k_{y}\right), \\
& \varepsilon_{k x y}=-4 t_{4} \sin k_{x} \sin k_{y}
\end{aligned}
$$

The intra-orbital components of the nearest-neighbour (NN) hopping integrals $t_{i j}^{\alpha \beta}$ are $\mathrm{t}_{x}^{11}=\mathrm{t}_{1}=-1$, and $\mathrm{t}_{x}^{22}=\mathrm{t}_{2}=1.3$. The components of next-nearest-neighbour ( $N N N$ ) hopping integrals, $\mathrm{t}_{i j}^{\prime \alpha \beta}$, are $t_{3}=t_{4}=-0.85$ Raghu et al. (2008). Throughout this paper, all the energies are measured in units of $\left|t_{1}\right|$. The carrier concentration is equal to 0.18 , which is a typical doping concentration in the iron-based superconductors Dubroka et al. (2008). 
The interaction term reads,

$$
H_{J-J^{\prime}}=J \sum_{<i j>\alpha \beta}\left(\vec{S}_{i \alpha} \cdot \vec{S}_{j \beta}-\frac{1}{4} n_{i \alpha} \cdot n_{j \beta}\right)+J^{\prime} \sum_{<<i j>>\alpha \beta}\left(\vec{S}_{i \alpha} \cdot \vec{S}_{j \beta}-\frac{1}{4} n_{i \alpha} \cdot n_{j \beta}\right)
$$

which contains a NN and a NNN antiferromagnetic spin couplings. Here $J$ and $J^{\prime}$ are the NN and the NNN spin coupling strengths, respectively. $\vec{S}_{i \alpha}$ is the spin operator of the electron in the $\alpha$-orbit at $\mathrm{R}_{i}$ and $n_{i \alpha}$ is the particle number operator. $\alpha, \beta(=1,2)$ are orbital index.

To explore the essence of the iron-pnictide superconductors and other multi-orbital superconductors, we discuss the $\mathrm{t}-\mathrm{t}^{\prime}-\mathrm{J}-\mathrm{J}^{\prime}$ model in three different correlation regimes:

\subsection{Weak correlation regime}

When the kinetic energy of the $d_{x z^{-}}$and $d_{y z}$-electrons is much larger than the Coulomb interaction, we adopt the conventional mean-field decoupling approach to study the superconducting pairing symmetry and its orbital dependence. This ansatz is applicable for many FeAs-based and other multi-orbital superconductors with metallic ground states.

Notice that the $d_{x z}$ and $d_{y z}$ orbits are spatially anisotropic, in other words, the intra-orbital hopping integral along the $\mathrm{x}$-direction is not equal to that along the $\mathrm{y}$-direction for each orbital, as one can see from $\left|t_{1}\right| \neq\left|t_{2}\right|$. Due to the asymmetry of the different directions in different orbits, the amplitude of the superconducting gap of the local pairing along the $\mathrm{x}$-direction may be not equal to that along the $y$-direction in each orbit. Thus, the single orbital d-wave or s-wave superconducting order parameter, in which the superconducting energy gap has 4-fold symmetry of rotational invariance in the xy plane, is not suitable for describing the pairing symmetry of the intra-orbital superconducting order parameters in this multi-orbital system. Considering all of the possible kinetic correlations and the superconducting pairings for the NN and NNN sites along different directions, we introduce the following order parameters,

$$
\begin{gathered}
P_{x / y}^{\alpha}=\left\langle c_{i \alpha \sigma}^{\dagger} c_{j \alpha \sigma}>, \quad(j=i \pm \hat{x} / \hat{y})\right. \\
P_{x y}^{1 / 2}=\left\langle c_{i 1 \sigma}^{\dagger} c_{j 2 \sigma}>, \quad(j=i \pm \hat{x} / \hat{y})\right. \\
P_{3}^{\alpha}=\left\langle c_{i \alpha \sigma}^{\dagger} c_{j \alpha \sigma}>, \quad(j=i \pm(\hat{x} \pm \hat{y}))\right. \\
P_{x y}^{3 / 4}=\left\langle c_{i 1 \sigma}^{\dagger} c_{j 2 \sigma}>, \quad(j=i \pm(\hat{x} \pm \hat{y}))\right. \\
\Delta_{x / y}^{1 \alpha}=J\left\langle c_{i \alpha \uparrow}^{\dagger} c_{j \alpha \downarrow}^{\dagger}>, \quad(j=i \pm \hat{x} / \hat{y})\right. \\
\Delta_{x \pm y}^{2 \alpha}=J^{\prime}\left\langle c_{i \alpha \uparrow}^{\dagger} c_{j \alpha \downarrow}^{\dagger}>, \quad(j=i \pm(\hat{x} \pm \hat{y})) .\right.
\end{gathered}
$$

Here $P_{x / y}^{\alpha}$ and $P_{x y}^{1 / 2}\left(P_{3}^{\alpha}\right.$ and $\left.P_{x y}^{3 / 4}\right)$ are the kinetic average of the NN (NNN) intra-orbital and inter-orbital hopping integrals. These terms could be decoupled within the framework of the mean-field approximationSeo et al. (2008). $\Delta_{x / y}^{1 \alpha}\left(\Delta_{x \pm y}^{2 \alpha}\right)$ is the mean-field amplitude of the local NN (NNN) pairing order parameter in the $\alpha$-orbit. The inter-orbital pairing parameter $\left\langle c_{i 1 \uparrow}^{\dagger} c_{j 2 \downarrow}^{\dagger}>\right.$ is very small, hence is neglected Seo et al. (2008).

With these parameters, one can decouple the interaction terms in Eq.(3) within the framework of the self-consistent mean-field approximation, and obtain the mean-field Hamiltonian, 


$$
\begin{array}{r}
H_{M F}=\sum_{k} \Psi(k)^{\dagger} A(k) \Psi(k)+\text { const. Here } \Psi(k)=\left(c_{k, 1, \uparrow}, c_{-k, 1, \downarrow}^{\dagger} c_{k, 2, \uparrow}, c_{-k, 2, \downarrow}^{\dagger}\right), \\
A(k)=\left(\begin{array}{cccc}
\epsilon_{k 1 \uparrow}-\mu & \Delta_{1}^{*}(k) & \epsilon_{k 12} & 0 \\
\Delta_{1}(k) & -\epsilon_{k 1 \downarrow}+\mu & 0 & -\epsilon_{k 12} \\
\epsilon_{k 12} & 0 & \epsilon_{k 2 \uparrow}-\mu & \Delta_{2}^{*}(k) \\
0 & -\epsilon_{k 12} & \Delta_{2}(k) & -\epsilon_{k 2 \downarrow}+\mu
\end{array}\right),
\end{array}
$$

and const is the collection of all the constant energy terms from the mean-field decoupling. The modified intra-orbital and inter-orbital kinetic energy reads,

$$
\begin{aligned}
\varepsilon_{k 1 \sigma}= & \varepsilon_{k x z}-2 J\left(P_{x}^{1} \cos k_{x}+P_{y}^{1} \cos k_{y}\right)-4 J^{\prime} P_{3}^{1} \cos k_{x} \cos k_{y} \\
& -4\left(J+J^{\prime}\right)\left(<n_{1 \sigma}>+<n_{2 \sigma}>\right) \\
\varepsilon_{k 2 \sigma}= & \varepsilon_{k y z}-2 J\left(P_{x}^{2} \cos k_{x}+P_{y}^{2} \cos k_{y}\right)-4 J^{\prime} P_{3}^{2} \cos k_{x} \cos k_{y} \\
& -4\left(J+J^{\prime}\right)\left(<n_{1 \sigma}>+<n_{2 \sigma}>\right) \\
\varepsilon_{k 12}= & \varepsilon_{k x y}-2 J\left(P_{x y}^{1} \cos k_{x}+P_{x y}^{2} \cos k_{y}\right) \\
& -4 J^{\prime}\left(P_{x y}^{3} \cos \left(k_{x}+k_{y}\right)+P_{x y}^{4} \cos \left(k_{x}-k_{y}\right)\right) .
\end{aligned}
$$

The superconducting order parameter $\Delta_{\alpha}(k)$ of each orbital channel in the momentum space is

$$
\begin{aligned}
\Delta_{\alpha}(k)= & -4\left(\Delta_{x}^{1 \alpha} \cos k_{x}+\Delta_{y}^{1 \alpha} \cos k_{y}\right) \\
& -4\left(\Delta_{x+y}^{2 \alpha} \cos \left(k_{x}+k_{y}\right)+\Delta_{x-y}^{2 \alpha} \cos \left(k_{x}-k_{y}\right)\right) \\
= & -4 \Delta_{x}^{1 \alpha}\left[\left(\cos \left(k_{x}\right) \pm \eta_{1 \alpha} \cos \left(k_{y}\right)\right)\right. \\
& \left.+\xi_{\alpha}\left(\cos \left(k_{x}+k_{y}\right) \pm \eta_{2 \alpha} \cos \left(k_{x}-k_{y}\right)\right)\right]
\end{aligned}
$$

Thus the superconducting pairing symmetry of the $\alpha$-orbit is determined by $\left(\cos \left(k_{x}\right) \pm \eta_{1 \alpha}\right.$ $\left.\cos \left(k_{y}\right)\right)+\xi_{\alpha}\left(\cos \left(k_{x}+k_{y}\right) \pm \eta_{2 \alpha} \cos \left(k_{x}-k_{y}\right)\right)$. Where $\xi_{\alpha}=\Delta_{x+y}^{2 \alpha} / \Delta_{x}^{1 \alpha}$; the anisotropic factors, $\eta_{1 \alpha}=\left|\Delta_{y}^{1 \alpha} / \Delta_{x}^{1 \alpha}\right|$ and $\eta_{2 \alpha}=\left|\Delta_{x-y}^{2 \alpha} / \Delta_{x+y}^{2 \alpha}\right|$, are positive. \pm denotes the relative phase of $\Delta_{y}^{1 \alpha}$ with respect to $\Delta_{x}^{1 \alpha}$ or $\Delta_{x-y}^{2 \alpha}$ to $\Delta_{x+y}^{2 \alpha}$.

To characterize the complicated superconducting order parameters in different parameter regions, we define the $S_{x^{2}+\eta y^{2}}$-wave or $d_{x^{2}-\eta y^{2}}$-wave as the pairing symmetry when $\Delta_{\alpha} \propto$ $\cos \left(k_{x}\right)+\eta \cos \left(k_{y}\right)$ or $\Delta_{\alpha} \propto \cos \left(k_{x}\right)-\eta \cos \left(k_{y}\right)$. It reduces to the conventional $S_{x^{2}+y^{2} \text {-wave }}$ or $d_{x^{2}-y^{2}}$-wave symmetry at $\eta=1$. We also define the $S_{\eta x^{2} y^{2}}$-wave or $d_{\eta x y}$-wave as the superconducting pairing symmetry when $\Delta_{\alpha} \propto \cos \left(k_{x}+k_{y}\right)+\eta \cos \left(k_{x}-k_{y}\right)$ or $\Delta_{\alpha} \propto$ $\left.\cos \left(k_{x}+k_{y}\right)-\eta \cos \left(k_{x}-k_{y}\right)\right)$. In this situation, it reduces to the familiar $S_{x^{2} y^{2}}$-wave or $d_{x y}$-wave symmetry at $\eta=1$.

Diagonalizing the matrix $A(k)$ by an unitary transformation $\mathrm{U}(\mathrm{k}), U(k)^{\dagger} A(k) U(k)$, and minimizing the free energy of the system with respect to these parameters in Eq.(5-7), one 
obtains the self-consistent equations,

$$
\begin{aligned}
n_{(1 / 2) \uparrow} & =\frac{1}{N} \sum_{k, \alpha} U_{(1 / 3) \alpha}^{*}(k) U_{(1 / 3) \alpha}(k) f\left(E_{\alpha}(k)\right) \\
n_{(1 / 2) \downarrow} & =\frac{1}{N} \sum_{k, \alpha} U_{(2 / 4) \alpha}^{*}(k) U_{(2 / 4) \alpha}(k)\left(1-f\left(E_{\alpha}(k)\right)\right) \\
P_{x / y}^{1 / 2} & =\frac{1}{N} \sum_{k, \alpha} \cos k_{x / y} U_{(1 / 3) \alpha}^{*}(k) U_{(1 / 3) \alpha}(k) f\left(E_{\alpha}(k)\right) \\
P_{3}^{1 / 2} & =\frac{1}{N} \sum_{k, \alpha} \cos k_{x} \cos k_{y} U_{(1 / 3) \alpha}^{*}(k) U_{(1 / 3) \alpha}(k) f\left(E_{\alpha}(k)\right) \\
P_{x y}^{1 / 2} & =\frac{1}{N} \sum_{k, \alpha} \cos k_{x / y} U_{1 \alpha}^{*}(k) U_{3 \alpha}(k) f\left(E_{\alpha}(k)\right) \\
P_{x y}^{3 / 4} & =\frac{1}{N} \sum_{k, \alpha} \cos \left(k_{x}+/-k_{y}\right) U_{1 \alpha}^{*}(k) U_{3 \alpha}(k) f\left(E_{\alpha}(k)\right) \\
\Delta_{x / y}^{1(1 / 2)} & =\frac{J}{N} \sum_{k, \alpha} \cos k_{x / y} U_{(1 / 3) \alpha}^{*}(k) U_{(2 / 4) \alpha}(k) f\left(E_{\alpha}(k)\right) \\
\Delta_{x \pm y}^{2(1 / 2)} & =\frac{J^{\prime}}{N} \sum_{k, \alpha} \cos \left(k_{x} \pm k_{y}\right) U_{(1 / 3) \alpha}^{*}(k) U_{(2 / 4) \alpha}(k) f\left(E_{\alpha}(k)\right)
\end{aligned}
$$

where $E_{\alpha}(k)$ is the Bogoliubov quasiparticle eigenvalues obtained from $\mathrm{H}_{M F}$, and $f(E)$ is the Fermi-Dirac distribution function, $\mathrm{f}(\mathrm{E})=1 /\left(1+\exp \left(E / k_{B} T\right)\right) . \mathrm{U}_{\alpha \beta}(\mathrm{k})$ denotes the $(\alpha, \beta)$ element of the $4 \times 4$ unitary matrix $\mathrm{U}(\mathrm{k})$.

With these self-consistent equations, we could obtain not only the groundstate phase diagram, but also the temperature dependence of the Fermi surfaces in normal state and the quasiparticle spectra in the normal and superconducting states. In fact, the intra-orbital hopping integral of the $d_{y z}$ orbit is symmetric with that of the $d_{y z}$ orbit under a coordinate transformation $(\mathrm{x}, \mathrm{y}, \mathrm{z}) \leftarrow(\mathrm{y}, \mathrm{x}, \mathrm{z})$. Due to this symmetry, the superconducting order parameters $\Delta_{2}(\mathrm{k})$ can be obtained from $\Delta_{1}(\mathrm{k})$ under the coordinate transformation. Therefore, we mainly focus on the properties of the superconducting order parameters $\Delta_{1}(\mathrm{k})$ in the first orbit $d_{x z}$. Nevertheless, the global superconducting pairing order parameter of the two-orbital $\mathrm{t}-\mathrm{t} \mathrm{t}^{\prime}-\mathrm{J}-\mathrm{J}^{\prime}$ model should be rotationally symmetric in the $x y$-plane, as we can see from the Hamiltonian Eq.(2).

Within the present scenario, we could obtain not only the groundstate phase diagram, but also the quasiparticle spectra in the normal and the superconducting states. The temperature dependence of the Fermi surface in normal state and that of the spin-lattice relaxation rate in the superconducting state can also be obtained. Among these quantities, the spin-lattice relaxation rate in the NMR experiment is expressed as Matano (2008):

$$
\begin{aligned}
\frac{T_{1 N}}{T_{1 s}}= & \frac{2}{k_{B} T} \iint N_{S}(E) N_{s}\left(E^{\prime}\right) f(E) \\
& \times\left[1-f\left(E^{\prime}\right) \delta\left(E-E^{\prime}\right) d E d E^{\prime}\right]
\end{aligned}
$$


Providing $1 / \mathrm{T}_{1 N}$ in the normal state satisfies the Korringa law, the spin lattice relaxation rate $1 / T_{1 s}$ becomes Xiang (2007): $1 / T_{1 s} \propto\left(k_{B} T\right) \cdot T_{1 N} / T_{1 s}$.

\subsection{Intermediate correlation regime}

When the kinetic energy of the conduction bands becomes small and is comparable with the Coulomb interaction, we need to consider the electronic correlation effect, as one sees in $\mathrm{FeTe}_{1-x} \mathrm{Se}_{x}$ and other superconductors. We utilize the Kotliar-Ruckenstein's slave boson approach for some FeAs-based and ruthenate superconductor with intermediate magnetic moments. To reflect the multi-orbital character of iron pnictides, we need to extend the single-orbital Kotliar-Ruckenstein's slave boson approach Kotliar et al. (1988) to the two-orbital Hubbard models for various configurations. In the multiorbital Hubbard model, a few of auxiliary boson field operators representing the possibilities of various electron occupations are introduced, such as $e, p, d, b, t, q$, which denote the possibilities of none, single, double, triplicate, quaternity occupations. With these auxiliary boson fields, an original fermion operator can be expressed as:

$$
\begin{aligned}
c_{i \alpha \sigma}^{\dagger} & =Q_{i \alpha \sigma}^{-\frac{1}{2}}\left(p_{i \alpha \sigma}^{\dagger} e_{i}+b_{i \alpha}^{\dagger} p_{i \alpha \sigma}+\sum_{\sigma^{\prime}} d_{i \sigma_{\alpha} \sigma_{\beta}^{\prime}}^{\dagger} p_{i \beta \sigma^{\prime}}+t_{i \alpha \sigma}^{\dagger} b_{i \beta}\right. \\
& \left.+\sum_{\sigma^{\prime}} t_{i \beta \sigma^{\prime}}^{\dagger} d_{i \bar{\sigma}_{\alpha} \sigma_{\beta}^{\prime}}+q_{i}^{\dagger} t_{i \alpha \bar{\sigma}}\right)\left(1-Q_{i \alpha \sigma}\right)^{-\frac{1}{2}} f_{i \alpha \sigma}^{\dagger}
\end{aligned}
$$

Here $f_{i \alpha \sigma}^{\dagger}$ is the new slaved fermion operator and $Q_{i \alpha \sigma}$ is an auxiliary particle number operator Kotliar et al. (1988). Projecting the original fermion operators into these boson field and fermion field operators, one could not only obtain an effective Hamiltonian, but also get the groundstate energy in the saddle point approximation with the normalization condition and the fermion number constraints Kotliar et al. (1988). Here we employ a generalized Lagrange multiplier method to enforce these constraint conditions, thus the interorbital hoppings and crystal field splitting can be treated on the same foot. The fermion occupation number is constrained with the penalty function method. To enforce the normalization condition, we have a boundary constrained condition:

$$
1 \geq \sum_{\alpha \sigma} p_{\alpha \sigma}^{2}+\sum_{\alpha} b_{\alpha}^{2}+\sum_{\alpha \sigma \sigma^{\prime}} d_{\alpha \sigma \sigma^{\prime}}^{2}+\sum_{\alpha \sigma} t_{\alpha \sigma}^{2}+q^{2}
$$

With these projections to the boson states, one can easily obtain an effective t-t $\mathrm{t}^{\prime} \mathrm{J}-\mathrm{J}^{\prime}$ model subjected to the normalization and fermion number constraints. Following the similar steps above in the weak correlation regime to decouple the spin exchange terms, one could obtain the self-consistent equations similar to Eq.(8) to get various supercoundcting groundstate properties in the intermediate correlated iron pnictides, such as FeSe/FeTe, etc.

\subsection{Strong correlation regime}

Once the Coulomb interaction is so large that double occupation is excluded on each site, we use the Barnes-Coleman's slave boson approach to discuss the pairing symmetry and orbital-dependent superconducting energy gaps, which is applicable for some FeAs-based superconductors and heavy fermion superconductors with significantly large magnetic moments in the parent phases. 
Within the slave-boson representation Barnes (1976); Coleman (1984), Eq.(4) is rewritten in terms of the projected fermion operators $f_{i m \sigma}^{\dagger}$ and $f_{i m \sigma}$, as well as the slave boson operators at each site, which rule out the double and multiple fermion occupancies. The constrained Hilbert space $(S)$ of each site $i$ is

$$
S_{i}=\{|1, \uparrow>,| 1, \downarrow>,|2, \uparrow>,| 2, \downarrow>, \mid 0,0>\}
$$

including the single-occupied states of the spin-up and spin-down in 1-orbit, and those in 2-orbit, together with the vacancy state, respectively. The present constrained spin-orbital formulation resembles to the 4 -fold degenerate state of pseudo-angular momentum $j=3 / 2$ proposed by Barnes Barnes (1976) and Coleman Coleman (1984), if we define $\mid f^{0}>=1$ $0,0>,\left|f^{1}: 3 / 2,-3 / 2>=\right| 1, \uparrow>,\left|f^{1}: 3 / 2,-1 / 2>=\right| 1, \downarrow>,\left|f^{1}: 3 / 2,1 / 2>=\right| 2, \uparrow>$, and $\left|f^{1}: 3 / 2,3 / 2\right\rangle=\mid 2, \downarrow>$. In this representation, $P c_{i m \sigma}^{\dagger} P=f_{i m \sigma}^{\dagger} b_{i}$. The boson operator $b_{i}^{+}$ creates an empty occupation state at the $i$ th site, and the fermion operator $f_{i m \sigma}^{\dagger}\left(f_{i m \sigma}\right)$ creates (annihilates) a slaved electron at site $i$ with the orbit $m$ and spin $\sigma(=\uparrow, \downarrow)$. After projecting the original fermion representation into the present boson representation, one could obtain an effective $t-\mathrm{t}^{\prime}-\mathrm{J}-\mathrm{J}^{\prime}$ model subjected to these constraints. Following the similar steps above in Sec.2.1 to decouple the spin exchange terms, one could readily solve the superconducting groundstate properties in strongly correlated $\mathrm{K}_{x} \mathrm{Fe}_{2-y} \mathrm{Se}_{2}$ compound.

\section{Pairing symmetry of multi-orbital iron-pnictide superconductors}

We present the pairing symmetry of the two-orbital $t-\mathrm{t}^{\prime}-\mathrm{J}-\mathrm{J}^{\prime}$ models for weak-correlated FeAs-based superconductors. The main numerical results in the weak correlation situation are addressed as follows. Also one could easily obtain the numerical results of the intermediate and strong correlation situations.

\subsection{Stability of unusual superconducting pairing symmetry}

First of all, we determine the stable ground state of the present two-orbital $t-t^{\prime}-J-J^{\prime}$ model with the electron filling $n=1-\delta$ in a square lattice through comparing the groundstate energies of various pairing-symmetric superconducting states: the isotropic $s$ wave, anisotropic $\mathrm{s}_{x^{2}-y^{2}}$ wave, $\mathrm{s}_{x^{2} y^{2}}$ wave, $\mathrm{d}_{x^{2}-y^{2}}$ wave, and $\mathrm{d}_{x^{2} y^{2}}$ wave, etc. By minimizing the groundstate energies of various candidates and finding a stablest state, we obtain phase diagrams of the systems for various parameters, such as the hopping integrals $t_{a b}$, the doping concentration $n$, the exchange parameters $J$ and $J^{\prime}$ and so on. Our numerical results show that in the superconducting phase of iron pnictides, the energy of the weakly anisotropic and nodeless $d_{x^{2}-y^{2}}+s_{x^{2} y^{2}}$-wave-like superconducting state is lower than those of the $s$-waves and $d$-wave states for most of the situations we investigated.

\subsection{Phase diagram of superconducting pairing symmetry}

In this subsection, we first obtain the phase diagrams and mark the pairing symmetry of each stable superconducting phase in the J-J' and t-J planes, and locate the most possible position of the pairing symmetry of iron-pnictide superconductors.

The $\mathrm{J}^{\prime}-\mathrm{J}$ phase diagram of the $\mathrm{t}-\mathrm{t}^{\prime}-\mathrm{J}-\mathrm{J}^{\prime}$ model at carrier concentration $\mathrm{x}=0.18$ is shown in Fig.1a. Different from Seo et al.'s phase diagram Seo et al. (2008), we obtain five stable phases in the present model. The first one is a normal phase in the small $\mathrm{J}$ and $\mathrm{J}$ region, denoted 


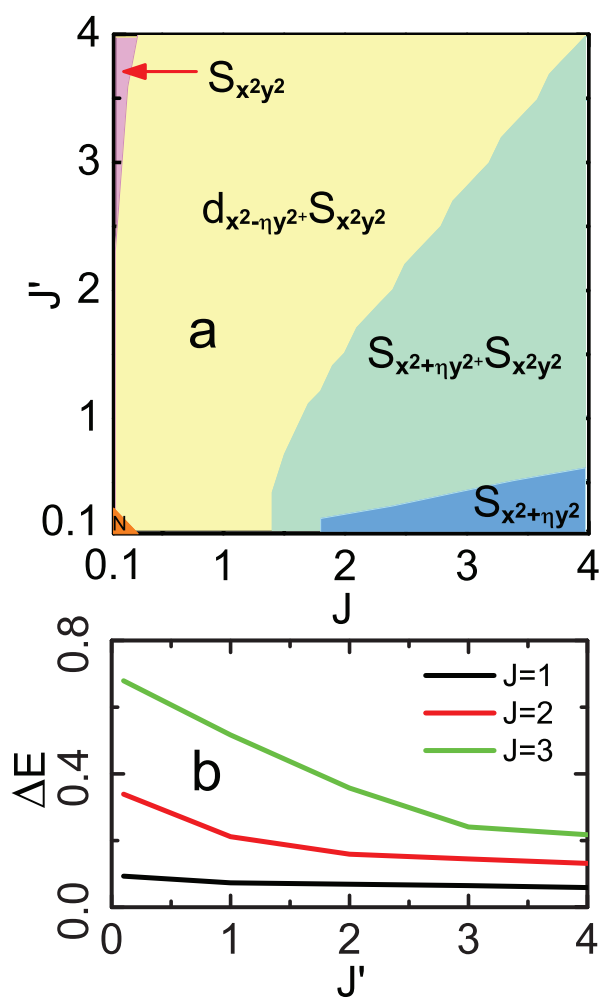

Fig. 1. (a) Superconducting phase diagram of the $t-t^{\prime}-J-J^{\prime}$ model for the $d_{x z}$-orbit at the carrier concentration $\mathrm{x}=0.18$. $\mathrm{N}$ denotes the normal state, the other four phases are superconductive with different pairing symmetries. (b) The energy difference $\Delta \mathrm{E}$ between Seo et al's Seo et al. (2008) and our ground states vs the NNN spin coupling $\mathrm{J}^{\prime}$ at different $\mathrm{J}, \mathrm{J}=1,2$ and 3, respectively.

by $\mathrm{N}$ in Fig.1a. Obviously, when the superexchange coupling $\mathrm{J}$ and $\mathrm{J}$ are too small to provide the pairing glue, the kinetic energy is dominant, and the electrons stay in the normal state, which is analogous to the single-orbital t-J model Kotliar et al. (1988). Among the four superconducting phases mediated through the spin fluctuations, a large NN spin coupling $\mathrm{J}$ and a small NNN spin coupling $\mathrm{J}^{\prime}$, or $\mathrm{J}>>\mathrm{J}^{\prime}$, favor the $S_{x^{2}+\eta y^{2}}$ (here and below $\eta_{1}=\eta$ ) superconducting phase with the gap $\Delta_{1}(\mathrm{k}) \propto \cos \left(k_{x}\right)+\eta \cos \left(k_{y}\right)$, where the pairing symmetry is the combination of the $S_{x^{2}+y^{2}}$-wave and the $d_{x^{2}-y^{2}}$-wave components, as seen the pink region in Fig.1a. The $S_{x^{2}+\eta y^{2}}$ symmetry arises from the major contribution of the NN spin coupling J term. The NNN spin coupling contributes very little to $\Delta_{1}(\mathrm{k})$ due to $\mathrm{J}>>\mathrm{J}^{\prime}$.

On the other hand, small NN spin coupling J and large NNN spin coupling $\mathrm{J}^{\prime}$ favor the $S_{x^{2} y^{2}}$ superconducting phase with the symmetry $\Delta_{1}(\mathrm{k}) \propto \cos \left(k_{x}+k_{y}\right)+\cos \left(k_{x}-k_{y}\right)$, as seen in the blue region in Fig.1a, which is mainly attributed to the NNN spin coupling. In this situation, $\Delta_{1}(\mathrm{k})$ is almost isotropic in the $x y$-plane due to the isotropy of the dominant 
NNN hopping integrals in the $x y$-plane. The superconducting order parameter becomes complicated when J and $\mathrm{J}^{\prime}$ compete with each other. As seen in Fig.1a, the pairing symmetry of the superconducting phase in the green region of Fig.1a is the combination of the $S_{x^{2}+\eta y^{2}}$ and the $S_{x^{2} y^{2}}$ components, and the symmetry of the superconducting phase in the yellow region of Fig.1a is the combination of the $d_{x^{2}-\eta y^{2}}$ and the $S_{x^{2} y^{2}}$ components.

It is interesting to ask in which region the realistic parameters of the iron pnictides fall. From the first-principles calculations, Ma et. al. suggested that $\mathrm{J} \approx \mathrm{J}^{\prime} \approx 0.05 \mathrm{eV} / \mathrm{S}^{2} \mathrm{Ma}$ et al. (2010), where $S$ is the spin of each Fe ion. When the hopping integral $\left|t_{1}\right| \approx 0.1 \sim 0.5 \mathrm{eV}$, such a set of parameters falls in the yellow region in Fig.1a, implying that the FeAs superconductors should have the $d_{x^{2}-\eta y^{2}}+S_{x^{2} y^{2}}$ pairing symmetry, and the anisotropic factor $\eta$ is not equal to 1. Also, some other authors suggested other parameters for the FeAs superconductors, for example, Seo et al.Seo et al. (2008) proposed that $\mathrm{J}=0.25$ and $\mathrm{J}^{\prime}=0.5$; and Si et al.Si et al. (2008) thought that $\mathrm{J}>\mathrm{J}^{\prime} / 2$. This shows that further effort is needed to obtain more accurate interaction parameters in iron pnictides.

We notice that Seo et al.'s J-J' parameters also falls in the yellow region in Fig.1a, i.e. the pairing symmetry is $d_{x^{2}-\eta y^{2}}+S_{x^{2} y^{2}}$ type, rather than the $\delta_{S} S_{x^{2} y^{2}} \pm \delta_{d} d_{x^{2}-y^{2}}$ type with $\eta=1$ (here $\delta_{S}$ and $\delta_{d}$ are the weights of the $S_{x^{2} y^{2}}$ wave and the $d_{x^{2}-y^{2}}$ wave component, respectively). In Fig.1b, we compare the groundstate energy difference between theirs and ours, and find that the groundstate energy in the present superconducting phase, $\mathrm{E}_{\eta}$, is lower than the $\mathrm{E}_{d}$ in Seo et al.'s paper Seo et al. (2008). Fig.1b shows the J' dependence of the groundstate energy difference, $\Delta \mathrm{E}=\mathrm{E}_{d}-\mathrm{E}_{\eta}$, between the two superconducting phases at different $\mathrm{J}$, here $\mathrm{E}_{d}$ and $\mathrm{E}_{\eta}$ are the energies of the $\delta_{s} S_{x^{2} y^{2}} \pm \delta_{d} d_{x^{2}-y^{2}}$ symmetric phase and of the symmetric phase in Fig. 1a, respectively. It is clear that in wide J-J' range, the $d_{x^{2}-\eta y^{2}}+S_{x^{2} y^{2}}$ phase is always more stable than the $\delta_{S} S_{x^{2} y^{2}} \pm \delta_{d} d_{x^{2}-y^{2}}$ phase. Thus $d_{x^{2}-\eta y^{2}}+S_{x^{2} y^{2}}$-wave is most likely the superconducting pairing symmetry in iron pnictide superconductors.

\subsection{T-dependence of fermi surface and superconducting energy gaps}

In the present situation with a weakly broken orbital symmetry, we find that two superconducting energy gaps synchronously approach zero as $\mathrm{T}$ is lifted to $\mathrm{T}_{c}$. To concretely discuss the properties of the superconducting state and the normal state, and compare the theory with the ARPES experimental results, in what follows, we focus on two sets of typical superexchange coupling parameters, Case I: $\mathrm{J}=0.3$ and $\mathrm{J}^{\prime}=0.7$, i.e., the $\mathrm{NN}$ spin coupling is weaker than the NNN coupling; and Case II: $\mathrm{J}=0.7$ and $\mathrm{J}^{\prime}=0.3$, i.e. the $\mathrm{NN}$ spin coupling is stronger than the NNN coupling. In both of the situations, the parameters fall in the yellow region in Fig.1a, so the superconducting pairing symmetries are $d_{x^{2}-\eta y^{2}}+S_{x^{2} y^{2}}$-wave. We present the temperature evolution of the Fermi surfaces in the normal state in Fig.2 for the case I with $\mathrm{J}=0.3$ and $\mathrm{J}^{\prime}=0.7$. The Fermi surface topology for the case II with $\mathrm{J}=0.7$ and $\mathrm{J}^{\prime}=0.3$ is almost identical to Fig.2, hence is not plotted. From Fig.2, one sees that in the large Brillouin zone (BZ) associated with the present $\mathrm{t}-\mathrm{t}^{\prime}-\mathrm{J}-\mathrm{J}^{\prime}$ model with one Fe atom per unit cell, there exist two hole-like Fermi sheets $\left(\alpha_{1}\right.$ and $\left.\alpha_{2}\right)$ around the $\Gamma$ point, and two electron-like Fermi sheets $\left(\beta_{1}\right.$ and $\left.\beta_{2}\right)$ around the $M$ point. This is in agreement with the ARPES experiment Ding et al. (2008) and consistent with the first-principle electronic structures calculations Mazin et al. (2008); Boeri et al. (2008); Cao et al. (2008); Singh et al. (2008); Lebègue et al. (2007); Ma et al. (2008). Interestingly, the hole-like Fermi sheets expand a little with increasing temperature; in contrast, the electron-like Fermi sheets shrink considerably. This indicates that the 


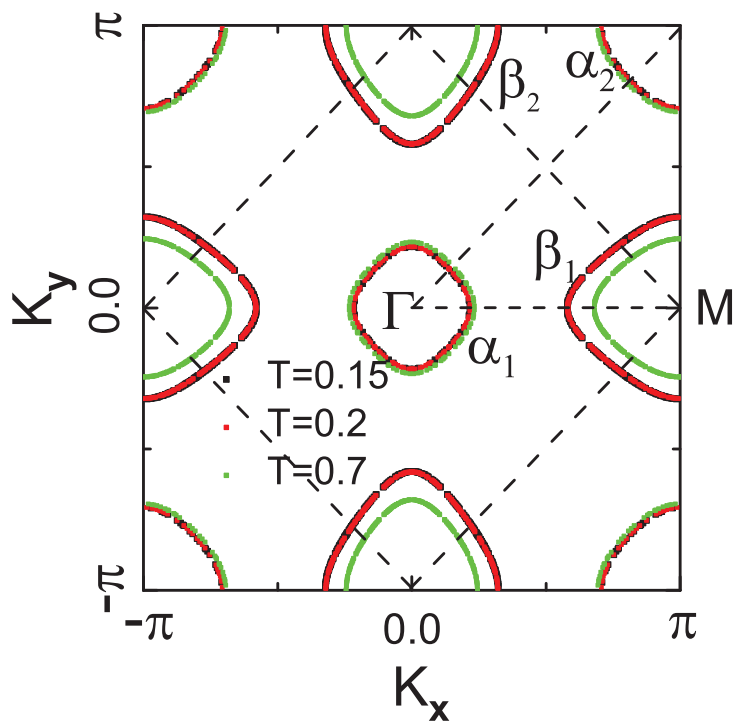

Fig. 2. The Fermi surface topology in the large Brillouin zone at the different temperatures: $\mathrm{T}=0.15$ (black), 0.2 (red) and 0.7 (green). Dashed square outlines the reduced Brillouin Zone. The theoretical parameters: $J=0.3, J^{\prime}=0.7$; the other parameters are the same as these in the Fig.1.

electron-like Fermi sheet may play a more important role in the low-energy processes in finite temperatures. This behavior arises from that the electronic thermal excitations increase with the lift of temperature, leading to the chemical potential decreasing with increasing $\mathrm{T}$. Thus the electron-like Fermi surface decreases and the hole-like Fermi surface increases with increasing $\mathrm{T}$.

Fig. 3 shows the temperature dependence of the superconducting energy gaps on the two hole-like Fermi sheets along the $\theta=0^{\circ}$ direction in the polar coordinate system for the two sets of parameters. With the increasing temperature, the two energy gaps decrease monotonously and vanish simultaneously, as observed in the ARPES experiments Ding et al. (2008). Obviously, the superconducting-state to normal-state transition is a second-order phase transition. For the case I, the magnitude of the energy gap on the small Fermi srface $\left(\alpha_{1}\right)$ is larger than that on the large Fermi surface $\left(\alpha_{2}\right)$ in the $\Gamma$ point, in agreement with the ARPES results Ding et al. (2008); Zhao et al. (2008). In contrast, for the case II, the magnitude of the gap on the small fermi surface $\left(\alpha_{1}\right)$ is smaller than that on large Fermi surface $\left(\alpha_{2}\right)$ in the $\Gamma$ point, which disagrees with the experiment Ding et al. (2008); Zhao et al. (2008). These indicate that the first set of parameters in Case I is more suitable for describing the FeAs superconductors.

From the present theoretical results in Fig.3, we find that in Case I, the ratios of the energy gaps to the transition temperature are $2 \Delta_{1} / k_{B} T_{c}=3.6$ for the large gap, and $2 \Delta_{2} / k_{B} T_{c}=2.9$ for the small gap, respectively. The ratio of the large energy gap around the small Fermi sheet to the small one around the large Fermi sheet gives rise to $\Delta_{1} / \Delta_{2}=1.25$. These theoretical 


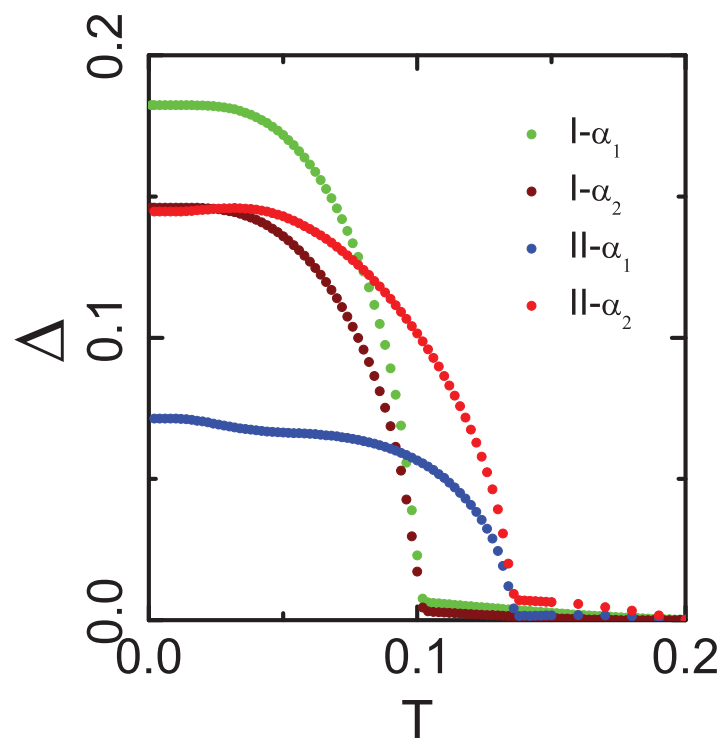

Fig. 3. Temperature dependence of the superconducting energy gaps near the small hole Fermi surface $\left(\alpha_{1}\right)$ and the large hole Fermi surface $\left(\alpha_{2}\right)$ around the $\Gamma$ point along the $\theta=0^{\circ}$ direction in the polar coordinate system. The doping concentration $x=0.18$. Theoretical parameters: Case I, $J=0.3, J^{\prime}=0.7$ (wine and green circles); and Case II, $J=0.7, J^{\prime}=0.3$ (red and blue circles).

results significantly deviate from the ARPES experimental data Ding et al. (2008). In Case II, $\Delta_{1} / \Delta_{2}=2$, in agreement with Ref.Ding et al. (2008), however, the ratios of these two gaps with respect to $T_{c}, 2 \Delta_{\alpha} / k_{B} T_{c}$, also strongly disagree with Ref.Ding et al. (2008). These facts demonstrate that there exist some essential shortages in the present $t-t^{\prime}-J-J^{\prime}$ model or in the self-consistent field method. One also notices that for Case II, the decline of the two superconducting energy gaps with the increasing temperature is not smooth, which comes from the fact that the different local pairing order parameters, $\Delta_{x / y}^{1 \alpha}$ and $\Delta_{x \pm y}^{2 \alpha}$, interplay with each other, reflecting the anisotropic pairing symmetry in Case II.

\subsection{Angle dependence of superconducting energy gaps}

In this section we present the dependence of each superconducting energy gap with the $\mathrm{d}+\mathrm{s}$-wave pairing on the orientational angle, and show that the anisotropy of the superconducting energy gaps crucially depends on the inter-orbital hopping and the ratio of $\mathrm{J}^{\prime} / \mathrm{J}$.

The ARPES experiment provides direct information about the quasiparticle spectra in normal state and the pairing symmetry of the superconducting energy gaps in the superconducting state. Here we present our theoretical results of the angle resolved energy gaps of the two orbits, and compare them with experimental observation. Fig. 4 shows the superconducting energy gap characters of the $\mathrm{t}-\mathrm{t}^{\prime}-\mathrm{J}-\mathrm{J}^{\prime}$ model for the two sets of the parameters in Case I and II. In 


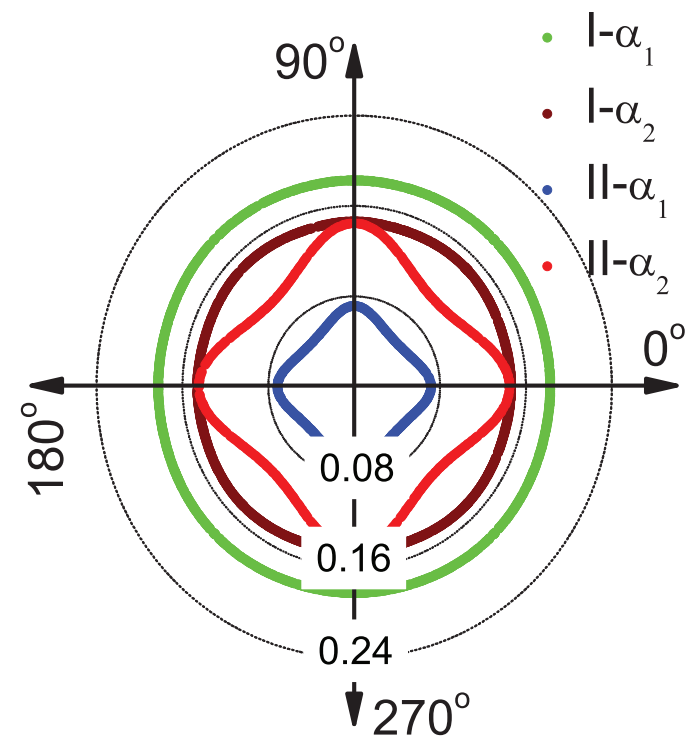

Fig. 4. The angle dependence of the superconducting energy gaps near the small hole Fermi surface $\left(\alpha_{1}\right)$ and the large hole Fermi surface $\left(\alpha_{2}\right)$ around the $\Gamma$ point in the polar coordinate system. The theoretical parameters are the same as these in Fig. 3 , Case I, $J=0.3, J^{\prime}=0.7$ (wine and green circles); and Case II, $J=0.7, J^{\prime}=0.3$ (red and blue circles).

both cases, two distinct gaps open on the hole-like Fermi sheets, $\alpha_{1}$ and $\alpha_{2}$, as seen in Fig.2. The presence of two different energy gaps demonstrates the nature of a multi-gap superconductor in the $t-t^{\prime}-J-J '$ model. Our results show that for the case I, the superconducting energy gap structure exhibits a nearly isotropic symmetry with invisible anisotropy, as seen in Fig.4. A large energy gap opens on the small hole Fermi sheet $\left(\alpha_{1}\right)$, and a small energy gap opens on the large hole Fermi sheet $\left(\alpha_{2}\right)$. For the case II, the angular dependence of the energy gaps is visible, exhibiting weak spatial anisotropy. The oscillation amplitude is about $16 \%$. However, the amplitudes of the superconducting energy gaps on the different Fermi surfaces, $\alpha_{1}$ and $\alpha_{2}$, are contrast to these in Case I, i.e., a small energy gap opens on the small Fermi surface sheet $\left(\alpha_{1}\right)$, and a large energy gap opens on the large hole fermi surface sheet $\left(\alpha_{2}\right)$.

One finds that in Case I, the anisotropy of the superconducting energy gaps is very weak, consistent with Zhao et al.'s Zhao et al. (2008) and Ding et al.'s Ding et al. (2008) ARPES data. In Case II, the superconducting energy gaps with about $16 \%$ anisotropy is in agreement with the ARPES experiment by Kondo et al. Kondo et al. (2008). Noticing that in Case II, such spatial anisotropy is still under the resolution of the ARPES experiment, hence does not conflict with Zhao et al.'s Zhao et al. (2008) and Ding et al.'s Ding et al. (2008) observation. It is the $d_{x^{2}-\eta y^{2}}+S_{x^{2} y^{2}}$-wave pairing symmetry that leads to weakly anisotropy and nodeless superconducting energy gap structures. Although the $d_{x^{2}-\eta y^{2}}$-wave pairing has nodes in the

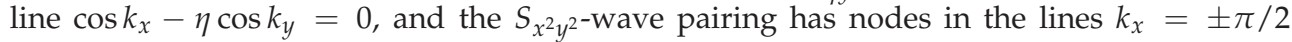
or $k_{y}=\pi / 2$. The mixed superconducting pairing symmetry, $d_{x^{2}-\eta y^{2}}+S_{x^{2} y^{2}}$, diminishes the 
nodes, so the system exhibits weakly anisotropic and nodeless s-wave-like energy gaps on the Fermi surface sheets.

\subsection{Spin-lattice relaxation rate in NMR}

In this subsection one can also obtain the theoretical spin-lattice relaxation data under different temperatures, especially the temperature-dependence of the Knight shift in iron-pnictide superconductors. We attribute the unusual T-dependence of the Knight shift to the multi-gap character.

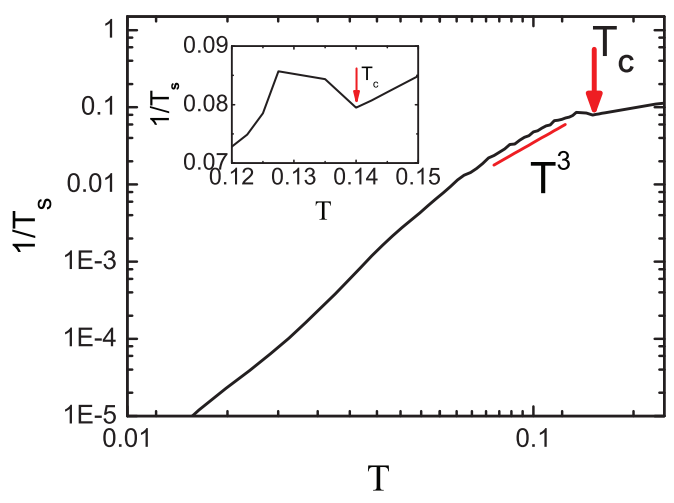

Fig. 5. Temperature dependence of the spin lattice relaxation rate in the $t-t^{\prime}-J-J^{\prime}$ model. The red arrow indicates the superconducting critical temperature $\mathrm{T}_{\mathcal{c}}$. The red line is the $\mathrm{T}^{3}$ law for comparison. Inset shows the detail near $\mathrm{T}_{\mathcal{C}}$. The theoretical parameters are the same as case II in Fig.2.

Although many experimental measurements, such as the Andreev reflection Chen et al. (2008), the exponential temperature dependence of the penetration depths and the ARPES Ding et al. (2008); Zhao et al. (2008), observed the nodeless gap function in the superconducting phase of $\mathrm{ReFeAsO}_{1-x} \mathrm{~F}_{x}$ and $\mathrm{Ba}_{1-x} \mathrm{~K}_{x} \mathrm{Fe}_{2} \mathrm{As}_{2}$ compounds, the line nodes in the superconducting energy gap was also suggested by the NMR experiment Matano (2008). The two characters in the NMR experiment supported the line nodes: lack of the coherence peak and the $T^{3}$ behavior in the nuclear spin-lattice relaxation rate, $1 / T_{1}$. Using the gap function obtained in this paper, we calculate the spin lattice relaxation rate $1 / T_{s}$, and the numerical result is shown in Fig. 5. We also plot the $T^{3}$ law (the red line) for a comparison. It is found that over a wide temperature range, the spin lattice relaxation rate in the present model can be fitted by the $T^{3}$ law, in agreement with the observation of the NMR experiments Matano (2008).

A small coherence peak appears around the critical transition temperature, as clearly seen in the inset of Fig.5. Experimentally, such a small coherence peak may be easily suppressed by the impurity effect or the antiferromagnetic spin fluctuations, similar to the situations in cuprates. This leads to the missing of the Hebel-Slichter coherence peak in the NMR experiment in iron pnictide SC. With decreasing temperature, one finds a drop in the spin lattice relaxation rate, $1 / T_{1}$, consistent with the observation of the NMR experiments Matano 
(2008). Such a behavior deviating from the $T^{3}$ law may be attributed from the multi-gap character of this system, and such a drop reflects the different energy gaps in different orbits. Surely, more meticulous studies are needed in the near future. We also notice that the extended $s_{ \pm}$energy gaps found by Parker etal. also can give the same NMR relaxation rate in superconducting pnictides Parker et al. (2008). Parish et al. Parish et al. (2008) suggested that the deviation of the $T^{3}$ law in the spin-lattice relaxation arises from the inter-band contribution.

\section{Comparison with other theories and experimental observations}

From the preceding discussions, we find that many unusual properties in the normal state and the superconductive phase of newly discovered FeAs compounds could be qualitatively interpreted in the two-orbital $\mathrm{t}-\mathrm{t}^{\prime}-\mathrm{J}-\mathrm{J}^{\prime}$ model, showing that to some extent, this model is a good approximation to describe iron pnictide superconductors. Within this scenario, the mixing pairing symmetry with $d_{x^{2}-\eta y^{2}}+S_{x^{2} y^{2}}$-wave contributes to the weakly anisotropic and nodeless energy gaps. Such a pairing symmetry assembles the characters of usual $d$-wave and $s$-wave, hence shares the properties of the usual $d$-wave superconductors, like cuprates, and the $s$-wave superconductor, such as $\mathrm{MgB}_{2}$. Nevertheless, to quantitatively compare the theoretical results with the experimental observation, more subtle band structures of the $\mathrm{t}-\mathrm{t}^{\prime}-\mathrm{J}-\mathrm{J}^{\prime}$ model are expected.

It is of interest that for sufficiently large NNN spin coupling $\mathrm{J}^{\prime}, S_{x^{2} y^{2}}$ is a dominating pairing state which is the same as the pairing symmetry obtained by Chubukov et alChubukov et al. (2008). It may seem strange that this intermediate coupling theory based upon the proximity to a Mott transition has essentially the same pairing solutions as the Fermi-liquid analysis of Ref.Mazin et al. (2009). But it is not surprising at all because the fermiology and the spin fluctuation wave vector (the structure of magnetic excitations in the reciprocal space) predetermine this symmetry, as is suggested by Mazin et al. (2009). There is, however, an important difference between our results and those of Chubukov et al Chubukov et al. (2008). In their case, pairing mechanism is due to the increase in the intra-band pairing hopping term, not necessarily due to spin-fluctuations that is the pairing mechanism in our analysis.

Also, one should keep in mind that a completely quantitative comparison between the theory and experiment is still difficult, since the present two-orbital $t-t^{\prime}-J-J^{\prime}$ model only describes the topology structure of the Fermi surfaces of the FeAs superconductors, but does not contain all the details of the Fermi surfaces and the band structures in iron pnictide compounds. On the other hand, in the realistic material, the spin couplings, $J$ and $J^{\prime}$, might be a strong asymmetry Yin et al. (2008), which is not taken into account in the present $t-t^{\prime}-J-J^{\prime}$ model. Hence, we expect that the more elaborate tight-binding parameters and anisotropic coupling J-J' model will improve the present results in future studies. Also the present constrained mean-field approximation needs to be further improved.

In some FeAs-based superconductors, the weakly anisotropic orbital symmetry makes it very difficult to distinguish which orbitals are involved in the formation of the superconducting state. To further uncover the orbital dependence of the superconducting energy gaps, we study the superconducting properties of a highly anisotropic two-orbital t-J model in the strong correlation regime. We study how the phase diagram evolves with the band asymmetric factor $R=t_{22} / t_{11}$, and the numerical result is shown in Fig. 6 in the 
strongly correlation regime. Note that we consider only the nearest-neighbor hopping on a square lattice. It is found that at $n=1.98$, the difference between $\Delta_{1}$ and $\Delta_{2}$ increases with $R$ deviating from the unity. The superconducting order parameters exhibit different behaviors: $\Delta_{2}$ monotonously increases and almost saturates as $\mathrm{R}<R_{c} \approx 0.6$; however, $\Delta_{1}$ monotonously decreases and vanishes at $R_{c}$, indicating the appearance of an orbital dependent superconducting phase, where the superconducting gap in orbit 2 exponentially approaches zero, and the energy gap in orbit remains finite. As the doping concentration increases to $n=1.95$ in Fig.6b, the two superconducting order parameters behave similarly to Fig.6a. Finally, the TGSC-intermediate superconducting phase transition occurs at $R_{c} \approx 0.7$. Obviously, with the decrease of $R$, the bandwidth of 2-orbit considerably shrinks and the pairing coupling of the orbit-2 electrons significantly deviates from that of 1-orbit. Thus, the orbital-dependent intermediate superconducting phase easily occurs when the symmetry of the orbital hopping is broken.

With the increase of the hopping integral asymmetry, the bandwidth of the 2-orbit becomes narrower and narrower, and more and more orbit-2 electrons transfer to orbit-1, hence the amplitude of the superconducting order parameter of 2-orbit gradually decreases to zero. In the same time, the superconducting order parameter of 1-orbit increases. The system enters

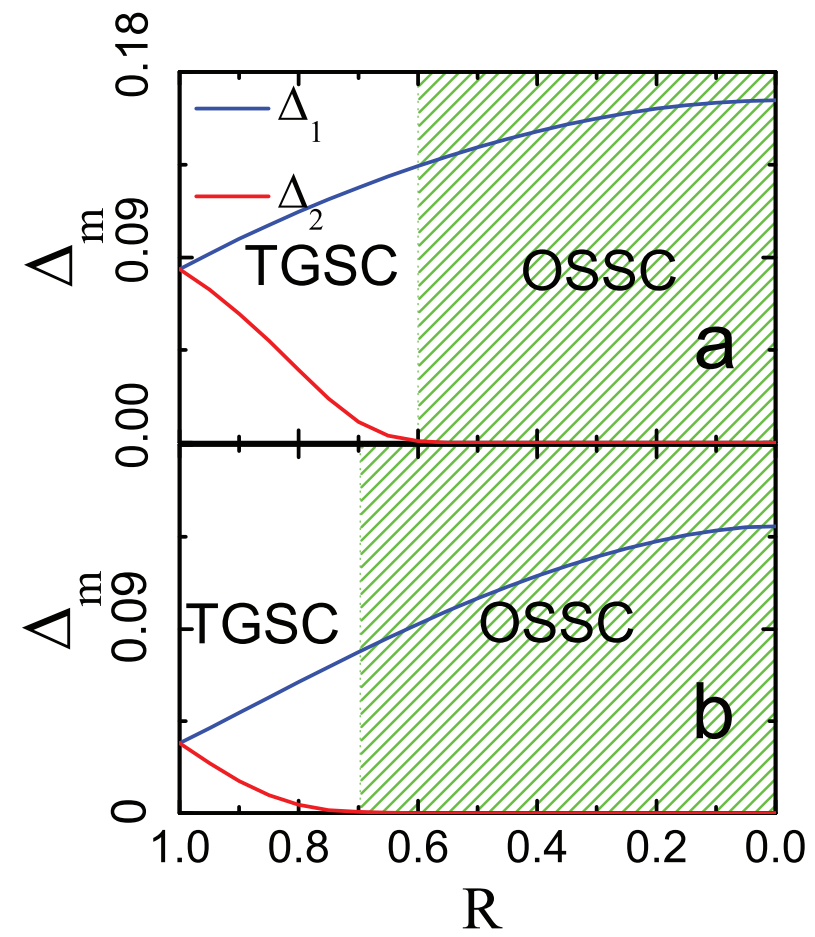

Fig. 6. (Color online) Dependence of the superconducting order parameters $\Delta_{m}(\mathrm{~m}=1,2)$ on the level splitting $E_{\Delta}$ for (a) $R=1$ and $\delta=0.02$, and (b) $R=0.8$ and $\delta=0.02$, respectively. Here TGSC and OSSC denote the two-gap and orbital dependent superconducting phases, respectively 
the orbital dependent superconducting regime, and the orbital dependent superconducting phase is more robust with the deviation of $\mathrm{R}$ from the unity, as we see in Fig.6b. As one expects, when the hopping integral ratio $\mathrm{R}$ is larger than the unity, the behavior of $\Delta_{1}$ is inter-changed with that of $\Delta_{2}$. The properties in the system with $R$ are analogous to those with $1 / R$ in the absence of the crystalline field splitting.

\section{Remarks and conclusions}

We notice the profound difference of the superconducting properties between cuprates and iron pnictides. Comparing with the copper-based superconductors with a 4 -fold rotational symmetry, the inequivalence between the $x$ - and $y$-direction of the orbit $\mathrm{d}_{x z / y z}$ in iron pnictides results in the anisotropic factor, $\eta$, and leads to a distinct pairing symmetry. In the present iron pnictide superconductors, the NNN spin coupling contributes an important role to the $S_{x^{2} y^{2}}$ pairing symmetry. Further, the multi-orbital character also contributes two weakly anisotropic and nodeless energy gaps, significantly different from the single energy gap in the cuprate superconductors.

Strong next-nearest-neighbour coupling and inter-orbital hopping in iron-pnictide superconductors favor a weak anisotropic and nodeless $d+s$ wave symmetry. From Eq.(3), one could see that the NN interaction J favors the order parameters $\Delta_{x / y}^{1 \alpha}$ and the NNN interaction $\mathrm{J}^{\prime}$ favors $\Delta_{x \pm y}^{2 \alpha}$. Thus, when the NN interaction J is dominant in the system, the local superconducting order parameters $\Delta_{x / y}^{1 \alpha}$ become a dominant term in Eq.(5); and when the NNN interaction $\mathrm{J}^{\prime}$ is considerably larger than $\mathrm{J}$, the local superconducting order parameters $\Delta_{x \pm y}^{2 \alpha}$ become dominant in Eq.(5).

In summary, our results have shown that many properties observed in iron-based superconductors could be comprehensively understood in the present model qualitatively. In the reasonable physical parameters region of $\mathrm{LaFeAsO}_{1-x} \mathrm{~F}_{x}$, the pairing symmetry of the model is nearly isotropic and nodeless $d_{x^{2}-\eta y^{2}}+S_{x^{2} y^{2}}$-wave, mainly originating from the Fermi surface topology and the spin fluctuation in these systems, which is in agreement with the observation of ARPES and the NMR experiments in ironpnictide superconductors.

\section{References}

Kamihara Y., Watanabe T., Hirano M. and Hosono H. (2008). Iron-based layered superconductor $\mathrm{La}\left[\mathrm{O}_{1-x} \mathrm{~F}_{x}\right] \mathrm{Fe} A s(\mathrm{x}=0.05-0.12)$ with $\mathrm{Tc}=26 \mathrm{~K}$, J. Am. Chem. Soc., Vol. 130, 3296-3297, ISSN 0002-7863.

Chen X. H., Wu T., Wu G., Liu R. H., Chen H., and Fang D. F. (2008). Superconductivity at 43 $\mathrm{K}$ in SmFeAsO ${ }_{1-x} \mathrm{~F}_{x}$. Nature, Vol. 453, 761-762, ISSN 0028-0836.

Chen G. F., Li Z., Li G., Zhou J., Wu D., Dong J., Hu W. Z., Zheng P., Chen Z. J., Luo J. L., and Wang N. L. (2008). Superconducting properties of Fe-based layered superconductor $\mathrm{LaO}_{0.9} \mathrm{~F}_{0.1}$ FeAs. Phys. Rev. Lett., Vol. 101, 057007, ISSN 0031-9007.

Wu G., Xie Y. L., Chen H., Zhong M., Liu R. H., Shi B. C., Li Q. J., Wang X. F., Wu T., Yan Y. J., Ying J. J., Chen X. H. (2009). Superconductivity at $56 \mathrm{~K}$ in samarium-doped SrFeAsF. J. Phys.: Condens. Matter, Vol. 21, 142203, ISSN 0953-8984.

McMillan W. L. (1968). Transition Temperature of Strong-Coupled Superconductors. Phys. Rev., Vol. 167, 331, ISSN 0031-899X. 
Jaroszynski J., Hunte F., Balicas L., Jo Youn-jung, Raievi I., Gurevich A., Larbalestier D. C., Balakirev F. F., Fang L., Cheng P., Jia Y., and Wen H. H. (2008). Upper critical fields and thermally-activated transport of $\mathrm{NdFeAsO}_{0.7} \mathrm{~F}_{0.3}$ single crystal. Phys. Rev. B, Vol. 78, 174523, ISSN 1098-0121.

You W.-L., Gu S.-J., Tian G.-S., Lin H.-Q., (2008). Constrain on possible pairing symmetry in a two-orbital model of FeAs-based superconductors, arXiv:0807.1493.

Mazin I. I., Singh D. J., Johannes M. D. and Du M.H. (2008). Unconventional Superconductivity with a Sign Reversal in the Order Parameter of $\mathrm{LaFeAsO}_{1-x} \mathrm{~F}_{x}$. Phys. Rev. Lett., Vol. 101, 057003, ISSN 0031-9007.

Boeri L., Dolgov O. V., and Golubov A. A. (2008). Is $\mathrm{LaFeAsO}_{1-x} \mathrm{~F}_{x}$ an Electron-Phonon Superconductor? Phys. Rev. Lett., Vol. 101, 026403, ISSN 0031-9007.

Cao C., Hirschfeld P.J., and Cheng H. P. (2008). Proximity of antiferromagnetism and superconductivity in $\mathrm{LaFeAsO}_{1-x} \mathrm{~F}_{x}$ : Effective Hamiltonian from ab initio studies. Phys. Rev. B, Vol. 77, 220506(R), ISSN 1098-0121.

Ma F. J., Lu Z. Y., and Xiang T. (2010). Electronic structures of ternary iron arsenides $\mathrm{AFe}_{2} \mathrm{As}_{2}$ (A=Ba, Ca, or Sr). Front. Phys. China, Vol. 5, 150 , ISSN 2095-0470.

Zhang H. J., Xu G., Dai X., and Fang Z. (2009). Enhanced Orbital Degeneracy in Momentum Space for LaOFeAs. Chinese Phys. Lett., Vol. 26, 017401, ISSN 0256-307X.

Mu G., Zhu X. Y., Fang L., Shan L., Ren C. and Wen H. H. (2008). Nodal Gap in Fe-Based Layered Superconductor $\mathrm{LaO}_{0.9} \mathrm{~F}_{0.1-\delta}$ FeAs Probed by Specific Heat Measurements. Chinese Phys. Lett., Vol. 25, 2221, ISSN 0256-307X.

Millo O., Asulin I., Yuli O., Felner I., Ren Z. A., Shen X. L., Che G. C., and Zhao Z. X. (2008). Scanning tunneling spectroscopy of $\mathrm{SmFeAsO}_{0.85}$ : Possible evidence for d-wave order-parameter symmetry. Phys. Rev. B, Vol. 78, 092505, ISSN 1098-0121.

Wang Y. L., Shan L., Fang L., Cheng P., Ren C., Wen H. H. (2009). Multiple gaps in $\mathrm{SmFeAsO} \mathrm{A}_{0.9} \mathrm{~F}_{0.1}$ revealed by point-contact spectroscopy. Supercond. Sci. Technol., Vol. 22, 015018 , ISSN 0953-2048.

Wang F., Zhai H., Ran Y., Vishwanath A., and Lee D. H. (2009). Functional Renormalization-Group Study of the Pairing Symmetry and Pairing Mechanism of the FeAs-Based High-Temperature Superconductor. Phys. Rev. Lett., Vol. 102, 047005, ISSN 0031-9007.

Nomura T., Kim S. W., Kamihara Y., Hirano M., Sushko P. V., Kato K., Takata M., Shluger A. L., and Hosono H. (2008). Crystallographic phase transition and high-Tc superconductivity in LaFeAsO:F. Supercond. Sci. Technol., Vol. 21, 125028, ISSN 0953-2048.

Seo K., Bernevig B. A., and Hu J. P. (2008). Pairing Symmetry in a Two-Orbital Exchange Coupling Model of Oxypnictides. Phys. Rev. Lett., Vol. 101, 206404, ISSN 0031-9007.

Dai X., Fang Z., Zhou Y. and Zhang F. C. (2008). Even Parity, Orbital Singlet, and Spin Triplet Pairing for Superconducting $\mathrm{LaFeAsO}_{1-x} \mathrm{~F}_{x}$. Phys. Rev. Lett., Vol. 101, 057008, ISSN 0031-9007.

Lee P. A., and Wen X. G. (2008). Spin-triplet p-wave pairing in a three-orbital model for iron pnictide superconductors. Phys. Rev. B, Vol. 78, 144517, ISSN 1098-0121.

Kuroki K., Onari S., Arita R., Usui H., Tanaka Y., Kontani H., and Aoki H. (2008). Unconventional Pairing Originating from the Disconnected Fermi Surfaces of Superconducting $\mathrm{LaFeAsO}_{1-x} \mathrm{~F}_{x}$. Phys. Rev. Lett., Vol. 101, 087004, ISSN 0031-9007.

Yan X.-W., Gao M., LU Z.-Y., Xiang T. (2011). Electronic and magnetic structures of ternary iron selenides $\mathrm{AFe}_{2} \mathrm{Se}_{2}$ (A=K, Cs, or Tl). Phys. Rev. B, Vol. 84, 054502, ISSN 1098-0121. 
Haule K., Shim J. H., and Kotliar G. (2008). Correlated Electronic Structure of $\mathrm{LaO}_{1-x} \mathrm{~F}_{x} \mathrm{FeAs}$. Phys. Rev. Lett., Vol. 100, 226402, ISSN 0031-9007.

Manousakis E., Ren J., Meng S, and Kaxiras E. (2008). Effective Hamiltonian for FeAs-based superconductors. Phys. Rev. B, Vol. 78, 205112, ISSN 1098-0121.

Raghu S., Qi X. L., Liu C. X., Scalapino D., Zhang S. C. (2008). Minimal two-band model of the superconducting iron oxypnictides. Phys. Rev. B, Vol. 77, 220503(R), ISSN 1098-0121.

Dubroka A., Kim K. W., Roessle M., Malik V. K., Liu R. H., Wu G., Chen X. H., Bernhard C. (2008). Superconducting Energy Gap and c-Axis Plasma Frequency of $(\mathrm{Nd}, \mathrm{Sm}) \mathrm{Fe} \mathrm{AsO}_{0.82} \mathrm{~F}_{0.18}$ Superconductors from Infrared Ellipsometry. Phys. Rev. Lett., Vol. 101, 097011, ISSN 0031-9007.

Matano K., Ren Z. A., Dong X. L., Sun L. L., Zhao Z. X., and Zheng Q. Q. (2008). Spin-singlet superconductivity with multiple gaps in $\mathrm{PrFeAsO}_{0} \cdot 89 \mathrm{~F}_{0} \cdot 11$ Europys. Lett., Vol. 83, 57001, ISSN 0295-5075.

Xiang T., "d-Wave Superconductors", pp226, Science Press Company, (Beijing 2007), ISBN 978-7-03-018920-2.

Kotliar G., and Liu J. L. (1988). Superexchange mechanism and d-wave superconductivity. Phys. Rev. B, Vol. 38, 5142, ISSN 1098-0121.

Barnes S. E.. (1976). New method for the Anderson model. J. Phys. F: Met. Phys., Vol. 6, 1375, ISSN 0305-4608.

Coleman P. (1984). New approach to the mixed-valence problem. Phys. Rev. B, Vol. 29, 3035-3044, ISSN 1098-0121.

Si Q. M., and Abrahams E. (2008). Strong Correlations and Magnetic Frustration in the High Tc Iron Pnictides. Phys. Rev. Lett., Vol. 101, 076401, ISSN 0031-9007.

Ding H., Richard P., Nakayama K., Sugawara K., Arakane T., Sekiba Y., Takayama A., Souma S., Sato T., Takahashi T., Wang Z., Dai X., Fang Z., Chen G. F., Luo J. L. and Wang N. L. (2008). Observation of Fermi-surfaceĺCdependent nodeless superconducting gaps in $\mathrm{Ba}_{0} .6 \mathrm{~K}_{0} .4 \mathrm{Fe}_{2} \mathrm{As}_{2}$. Europys. Lett., Vol. 83, 47001, ISSN 0295-5075.

Singh D., and Du M. H. (2008). Density Functional Study of $\mathrm{LaFeAsO}_{1-x} \mathrm{~F}_{x}$ : A Low Carrier Density Superconductor Near Itinerant Magnetism. Phys. Rev. Lett., Vol. 100, 237003, ISSN 0031-9007.

Lebègue S. (2007). Electronic structure and properties of the Fermi surface of the superconductor LaOFeP. Phys. Rev. B, Vol. 75, 035110, ISSN 1098-0121.

Ma F., and Lu Z. Y. (2008). Iron-based layered compound $\mathrm{LaFeAsO}$ is an antiferromagnetic semimetal. Phys. Rev. B, Vol. 77, 033111, ISSN 1098-0121.

Zhao L., Liu H. Y., Zhang W. T., Meng J. Q., Jia X. W., Liu G. D., Dong X. L., Chen G. F., Luo J. L., Wang N. L., Lu W., Wang G .L., Zhou Y., Zhu Y., Wang X. Y., Xu Z. Y., Chen C. T., and Zhou X. J. (2008). Multiple Nodeless Superconducting Gaps in $\left(\mathrm{Ba}_{0} \cdot 6 \mathrm{~K}_{0} \cdot 4\right) \mathrm{Fe}_{2} \mathrm{As}_{2}$ Superconductor from Angle-Resolved Photoemission Spectroscopy. Chinese Phys. Lett., Vol. 25, 4402-4405, ISSN 0256-307X.

Kondo T., Santander-Syro A. F., Copie O., Liu C., Tillman M. E., Mun E. D., Schmalian J., Bud'ko S. L., Tanatar M. A., Canfield P. C., Kaminski A. (2008). Momentum Dependence of the Superconducting Gap in $\mathrm{NdFeAsO}_{0.9} \mathrm{~F}_{0.1}$ Single Crystals Measured by Angle Resolved Photoemission Spectroscopy. Phys. Rev. Lett., Vol. 101, 147003, ISSN 0031-9007.

Chen T. Y., Tesanovic Z., Liu R. H., Chen X. H., and Chien C. L. (2008). Superconductivity at $43 \mathrm{~K}_{\text {in }} \mathrm{SmFeAsO}_{1-x} \mathrm{~F}_{x}$. Nature, Vol. 453, 1224-1227, ISSN 0028-0836. 
Parker D., Dolgov O. V., Korshunov M. M., Golubov A. A., and Mazin I. I. (2008). Extended $\mathrm{S}_{+-}$scenario for the nuclear spin-lattice relaxation rate in superconducting pnictides. Phys. Rev. B, Vol. 78, 134524, ISSN 1098-0121.

Parish M. M., Hu J. P., and Bernevig B. A. (2008). Experimental consequences of the s-wave $\cos (\mathrm{kx}) \cos (\mathrm{ky})$ superconductivity in the iron pnictides. Phys. Rev. B, Vol. 78, 144514, ISSN 1098-0121.

Chubukov A. V., Efremov D. V., and Eremin I. (2008). Magnetism, superconductivity, and pairing symmetry in iron-based superconductors. Phys. Rev. B, Vol. 78, 134512, ISSN 1098-0121.

Mazin I. I., and Schmalian J. (2009). Pairing symmetry and pairing state in ferropnictides: Theoretical overview. Physica C, Vol. 469, 614, ISSN 0921-4534.

Yin Z. P., Lebílgue S., Han M. J., Neal B. P., Savrasov S. Y., and Pickett W. E. (2008). Electron-Hole Symmetry and Magnetic Coupling in Antiferromagnetic LaFeAsO. Phys. Rev. Lett., Vol. 101, 047001, ISSN 0031-9007. 


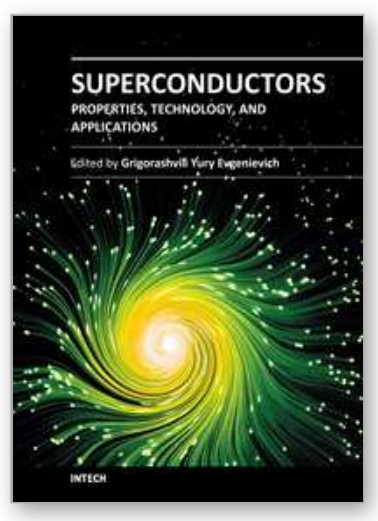

\author{
Superconductors - Properties, Technology, and Applications \\ Edited by Dr. Yury Grigorashvili
}

ISBN 978-953-51-0545-9

Hard cover, 436 pages

Publisher InTech

Published online 20, April, 2012

Published in print edition April, 2012

Book "Superconductors - Properties, Technology, and Applications" gives an overview of major problems encountered in this field of study. Most of the material presented in this book is the result of authors' own research that has been carried out over a long period of time. A number of chapters thoroughly describe the fundamental electrical and structural properties of the superconductors as well as the methods researching those properties. The sourcebook comprehensively covers the advanced techniques and concepts of superconductivity. It's intended for a wide range of readers.

\title{
How to reference
}

In order to correctly reference this scholarly work, feel free to copy and paste the following:

Liang-Jian Zou and Feng Lu (2012). Pairing Symmetry and Multiple Energy Gaps in Multi-Orbital Iron-Pnictide Superconductors, Superconductors - Properties, Technology, and Applications, Dr. Yury Grigorashvili (Ed.), ISBN: 978-953-51-0545-9, InTech, Available from: http://www.intechopen.com/books/superconductorsproperties-technology-and-applications/pairing-symmetry-and-multiple-energy-gaps-in-multi-orbitalsuperconductors

\section{INTECH}

open science | open minds

\section{InTech Europe}

University Campus STeP Ri

Slavka Krautzeka 83/A

51000 Rijeka, Croatia

Phone: +385 (51) 770447

Fax: +385 (51) 686166

www.intechopen.com

\section{InTech China}

Unit 405, Office Block, Hotel Equatorial Shanghai

No.65, Yan An Road (West), Shanghai, 200040, China

中国上海市延安西路65号上海国际贵都大饭店办公楼405单元

Phone: +86-21-62489820

Fax: +86-21-62489821 
(C) 2012 The Author(s). Licensee IntechOpen. This is an open access article distributed under the terms of the Creative Commons Attribution 3.0 License, which permits unrestricted use, distribution, and reproduction in any medium, provided the original work is properly cited. 